\title{
LOS SUJETOS DEL DESARROLLO RURAL EN ARGENTINA DE AGRICULTORES FAMILIARES A EMPRENDEDORES
}

\section{Marcos Andrés Urcola y María Elena Nogueira}

\section{Resumen}

El propósito central de este escrito es indagar acerca de los sujetos del desarrollo rural y las políticas públicas dirigidas a los agricultores familiares, focalizando en aquellos aspectos que manifiestan cambios en Argentina entre 2015 y 2019. En el contexto de reducción de ciertas capacidades estatales, el emprendedurismo se presenta como una propuesta conceptual y empírica que disputa sentido político y reorienta las políticas públicas, con instrumentos que tornan difusos sus atributos específicos. El trabajo se desarrolla desde una estrategia metodológica cualitativa centrada en el análisis de los discursos oficiales y el registro de las transformaciones de las estructuras estatales y programas destinados a la agricultura familiar.

Palabras clave: agricultura familiar, emprendedurismo, desarrollo rural, Argentina.

\begin{abstract}
TThe subjects of rural development in Argentina. From family farms to entrepreneurs

The central purpose of the present paper is to inquire about the subjects of rural development and the particular public policies connected to family farm, trying to focus on those aspects that show changes in their structure in Argentina between 2015 and 2019. In a context of reducing certain state capacities, we argue that entrepreneurship is presented as a conceptual and empirical proposal that is disputing the political meaning of family farm, redirecting public policies with instruments and programs that redefine their specific attributes. For this paper, we specially used a qualitative methodological strategy focused on the analysis of official discourses, the transformations of state structures and programs to family farm.
\end{abstract}

Keywords: family farm, entrepreneurship, rural development, Argentina.

Marcos Andrés Urcola: Investigador adjunto del Consejo Nacional de Investigaciones Científicas y Técnicas (CONICET) - Instituto de Investigaciones, Facultad de Ciencia Política y Relaciones Internacionales, Universidad Nacional de Rosario (UNR), Argentina.

ORCID iD: 0000-0003-0943-9354

Email:murcola@hotmail.com

María Elena Nogueira: Investigadora adjunta del Consejo Nacional de Investigaciones Científicas y Técnicas (CONICET) - Instituto de Investigaciones, Facultad de Ciencia Política y Relaciones Internacionales, Universidad Nacional de Rosario (UNR), Argentina.

ORCID iD: 0000-0001-6820-5721

Email: mariaelenanogueira@gmail.com 


\section{Introducción}

\section{Contexto y presentación de la problemática}

Los temas vinculados con el desarrollo rural y la agricultura familiar son objeto de discusión desde hace algunos años en América Latina. Históricamente, el interés por la temática del desarrollo rural identificaba como problemática casi única la "salida" de la pobreza de los pobladores rurales y los pequeños productores, a los que luego se identificaría como agricultores familiares. En este texto, nuestra pregunta se vincula con quiénes son los sujetos del desarrollo. Así como han variado las miradas sobre el desarrollo rural, tanto en sus aspectos teóricos como en aquellos más vinculados a sus modos de intervención, también lo han hecho los sujetos que dan cuenta de tal desarrollo. Para el logro de sus objetivos, cada programa o política específica presenta un recorte de la realidad, seleccionando y tipificando los sujetos destinatarios de sus acciones, bajo diversos supuestos teórico-metodológicos que guían su ejecución (Urcola, 2016). De este modo, la pregunta por los destinatarios de las acciones de desarrollo rural nos introduce en el debate empírico y teórico sobre la construcción de los sujetos de las políticas públicas en contextos específicos de intervención.

En América Latina, la estructura agraria se ha diferenciado tradicionalmente en dos tipos básicos de unidades productivas: las empresas agropecuarias o latifundios y las unidades campesinas o minifundistas. No obstante, desde mediados de la década del noventa y principios del nuevo milenio, en el interior de ambos grupos o como intersección de estos, comienza a identificarse en ámbitos académicos (universidades, centros de estudios, etcétera) y de gestión (organismos internacionales, Estados, gobiernos, etcétera) un universo de actores bajo el rótulo agricultores familiares. ${ }^{1}$

\footnotetext{
La agricultura familiar es una categoría acuñada inicialmente por científicos sociales europeos que evoca al modelo agrícola francés de la segunda posguerra. Dicha categoría fue incorporada desde la década del noventa por sindicatos y organizaciones de desarrollo rural en Brasil, en un contexto de descentralización y retracción del Estado y de organización territorial de los procesos económicos en América Latina. A pesar de estos antecedentes, recién en la década del 2000 el concepto comenzó a utilizarse en forma generalizada en los países de la región, a partir del impulso generado por informes y estudios de organismos internacionales, que comenzaron a incorporar en sus agendas los temas de la pobreza rural y la seguridad alimentaria vinculados a la producción de tipo familiar (Salcedo, De la O y Guzmán, 2014).
} 
Si bien hay cierto consenso en la idea simplificada de que el agricultor familiar es todo aquel sujeto que vive en el medio rural y trabaja, en principio, en la actividad agrícola junto con su familia, tanto investigadores como quienes elaboran políticas de desarrollo rural no concuerdan en una definición y esta difiere en forma considerable según la región o el país de referencia (Salcedo, De la O y Guzmán, 2014). ¿Qué implica ser un agricultor familiar para una política pública sectorial hoy? Esto refiere a una actualidad de gran variabilidad que nos interesa analizar, con especial interés en el caso argentino.

\section{El caso de Argentina}

En Argentina, la construcción de una agenda asociada al desarrollo rural y la agricultura familiar no ha sido habitual en el marco de las políticas destinadas al medio rural, y mucho menos en espacios estatales de gestión. Si bien el país tiene una larga tradición de estudios de carácter académico que sí han trabajado estas cuestiones, no habían sido abordadas, globalmente, en términos de políticas públicas hasta 2004, aproximadamente. En este sentido, la existencia de una política de desarrollo rural y agricultura familiar resulta más una excepción que una constante.

Para mencionar brevemente los contextos de esta excepcionalidad en Argentina puede indicarse que, primero, en la década del noventa, las acciones de los programas de desarrollo rural se centraban en la pequeña producción (minifundista) y en los pobres rurales (concebidos como sujetos de asistencia esencialmente); luego, en la década del 2000, una jerarquización institucional de la temática del desarrollo rural (con agencias estatales específicas al interior del Ministerio de Agricultura, Ganadería y Pesca de la Nación) ${ }^{2}$ identificó a la agricultura familiar como un sujeto económico relevante para llevar adelante procesos de desarrollo de los territorios rurales y como un sujeto político con derechos ciudadanos y demandas específicas que debían

2 En 2009 se creó el Ministerio de Agricultura, Ganadería y Pesca (MAGyP) y, en su interior, la Secretaría de Desarrollo Rural y Agricultura Familiar y la Subsecretaria de Agricultura Familiar (que en 2014 se elevó al rango de Secretaría). También en 2009, se creó al interior del MAGyP la Unidad para el Cambio Rural (UCAR), que aglutina a todos los programas de desarrollo rural con financiamiento internacional. Otro hito institucional de relevancia lo constituye la creación del Centro de Investigación y Desarrollo Tecnológico para la Pequeña Agricultura Familiar (CIPAF) del Instituto Nacional de Tecnología Agropecuaria (INTA) y la sanción, en 2014, de la Ley 27.118 de Reparación Histórica de la Agricultura Familiar para la Construcción de una Nueva Ruralidad en la Argentina. Una lectura a tener en cuenta para el análisis de este proceso en forma específica es Bertoni y Soverna (2015). Debe considerarse, además, la importancia del contexto regional que permitió, entre otras variables, la apertura e inclusión de problemáticas vinculadas con el desarrollo rural y la agricultura familiar en las agendas de gobierno. En forma previa a la definición de las estructuras estatales mencionadas, un hito insoslayable resulta la Reunión Especializada en Agricultura Familiar (REAF) del Mercosur, que habilitó regionalmente la discusión sobre estos temas. Al respecto, véase Lattuada, Nogueira y Urcola (2015). 
ser atendidas. Finalmente, en la actualidad (entre 2015 y 2019), si bien se sigue sosteniendo la formalidad de la institucionalidad antes construida, se observa una reinterpretación de los sujetos comprendidos en el universo de la agricultura familiar, planteando una vuelta a la identificación de estos sujetos con los pobladores rurales pobres y vulnerables de la década del noventa, o con sujetos con cualidades de innovación y emprendedurismo, ${ }^{3}$ cuyas lógicas los aproximan a conductas empresariales de las que antes, en buena medida, pretendían diferenciarse. ${ }^{4}$

A partir de 2015, coincidiendo con el cambio de gobierno a nivel nacional, ${ }^{5}$ se observan una serie de modificaciones de las estructuras estatales vinculadas al desarrollo rural y la agricultura familiar (desfinanciamiento y desjerarquización institucional de la agencias estatales) que están siendo acompañadas, desde el plano discursivo y conceptual, por una serie de redefiniciones sobre los sujetos destinatarios de las políticas públicas y la direccionalidad de estas, que merece nuestra atención analítica.

\section{Objetivos e hipótesis de trabajo}

Partimos del supuesto de que el contexto nacional (y regional $)^{6}$ de reducción de ciertas capacidades estatales está afectando las estructuras institucionales construidas en Argentina entre 2004 y 2014. Sostenemos que el emprendedurismo y los emprendedores rurales se presentan como una propuesta empírica y conceptual que disputa el sentido político de la agricultura familiar (y de otros actores de la economía popular) y reorienta las políticas públicas dirigidas al sector, con instrumentos y programas que redefinen o tornan difusos sus atributos específicos.

Con este telón de fondo, que incluye cuestiones conceptuales y empíricas, este escrito responde a la necesidad de indagar acerca de los sujetos del desarrollo rural y las políticas públicas, tratando de focalizar en aquellos

3 Si bien el término "emprendedurismo" no se encuentra reconocido por la Real Academia Española, es de uso frecuente en la literatura específica y en el vocabulario de las fuentes institucionales y periodísticas consultadas.

4 Téngase en cuenta que, en esencia, la agricultura familiar se ha presentado como una categoría que ha intentado aglutinar a los actores cuyas lógicas de acción económica, social y política se diferencian de las de los agentes agrarios empresariales o agroindustriales.

5 En diciembre de 2015 asumió la conducción del gobierno nacional la coalición política Cambiemos, integrada principalmente por dirigentes partidarios de Propuesta Republicana (PRO), referentes políticos de la Unión Cívica Radical (UCR) y de la Alianza Republicana (ARI). Este triunfo electoral puso fin al ciclo de doce años de gobierno de la coalición política de origen peronista denominada Frente para la Victoria.

6 En función de un análisis comparativo sobre las políticas de apoyo a la agricultura familiar de once países de América Latina durante los últimos cincuenta años, Sabourin (2018) sostiene que desde 2012 se está produciendo un proceso de erosión progresivo de las políticas para la agricultura familiar en términos de recursos asignados, ocupación de espacios institucionales y legitimación de sus demandas específicas. 
aspectos que manifiestan cambios en su consideración en el contexto actual (2015-2019) de la gestión estatal del desarrollo rural y la agricultura familiar en Argentina. Esta línea de trabajo forma parte de una investigación en curso que dialoga con estudios anteriores del equipo de investigación, sobre la configuración de los sujetos asociados a la gestión estatal del desarrollo rural en Argentina (Lattuada, Nogueira y Urcola, 2015).

El artículo se sostiene en una estrategia metodológica cualitativa centrada en el análisis de los discursos oficiales y el registro de las transformaciones de las estructuras estatales y los programas destinados al desarrollo de la agricultura familiar en el país. Sus fuentes principales han sido: información institucional, de prensa y entrevistas en profundidad a personal técnico de las agencias estatales de desarrollo rural. ${ }^{7}$

El presente artículo se compone de tres apartados y unas reflexiones finales. A esta introducción, le siguen un apartado de carácter teórico-conceptual en el que establecemos las claves analíticas que nos ayudan a comprender el nuevo contexto: la articulación conceptual entre agricultura familiar y los enfoques de desarrollo rural (que hacen de puente con nuestros estudios anteriores); el rastreo analítico de la noción de emprendedurismo y las discusiones que a su alrededor se establecen en el marco de las políticas públicas de desarrollo; y la búsqueda de enfoques teóricos que permitan la interpretación de estos fenómenos discursivos y empíricos a nivel de las políticas públicas. El siguiente apartado avanza en el análisis empírico de la penetración discursiva del emprendedurismo en el marco de las acciones del desarrollo rural actual en Argentina. Para ello, se describen algunas de las significativas modificaciones acontecidas en las áreas de gestión estatal destinadas al desarrollo rural y la agricultura familiar, desde 2015 a 2019; se examinan específicamente algunos programas de fomento de la cultura emprendedora y se ofrece un análisis marcando las disputas simbólicas e institucionales relacionadas con los sujetos destinatarios de las políticas públicas sectoriales y el modelo de participación e integración social que promueven. Para finalizar, presentamos algunas reflexiones de cierre que recuperan los ejes centrales del escrito.

7 Entre los meses de abril de 2018 y marzo de 2019 se realizó un total de diez entrevistas semiestructuradas y en profundidad a referentes de los equipos técnicos nacionales y provinciales de la Secretaría de Agricultura Familiar, Coordinación y Desarrollo Territorial (ex Secretaría de Agricultura Familiar) y de la Dirección General de Programas y Proyectos Sectoriales y Especiales (ex Unidad para el Cambio Rural). Una versión preliminar de este trabajo fue presentada en el X Congreso de la Asociación Latinoamericana de Sociología Rural (ALASRU), realizado en Montevideo (Uruguay) entre el 25 y el 30 de noviembre de 2018. 


\section{Abordaje teórico-conceptual}

\section{Los sujetos del desarrollo rural en la Argentina del nuevo milenio}

El tema del desarrollo rural se instala definitivamente en el país en la década del noventa, cuando se diagnosticó que la mitad de las explotaciones agropecuarias del territorio desaparecerían (incluso las pampeanas), en las condiciones del nuevo modelo de paridad cambiaria, apertura y desregulación de la economía (Lattuada, Nogueira y Urcola, 2015). En el marco de los procesos de reforma estructural de los Estados de esa época, la problemática del desarrollo rural se instaló en la agenda pública argentina como parte del proceso de consolidación de un modelo que minimiza el rol del Estado en la sociedad, que desplaza las decisiones al mercado y propone programas asistenciales y compensatorios para quienes se encuentran condenados a la exclusión en el nuevo contexto de acumulación e integración a la economía mundial. Es importante resaltar que, en el marco de los programas elaborados durante esta década, la agricultura familiar era considerada dentro de las acciones de asistencia a la pobreza y, más precisamente, como un segmento de "beneficiarios" focalizado en los pequeños productores rurales pobres, a los que se debía asistir técnica y financieramente.

Hacia finales de la década del noventa y principios del 2000, se difundió en nuestra región un nuevo discurso vinculado con temas y acciones de desarrollo endógeno, gestión estratégica de ámbitos locales, participación y territorio (Manzanal, 2006; Lattuada, Nogueira y Urcola, 2015). Este enfoque es de gran importancia para la reconsideración de la agricultura familiar como sujeto del desarrollo y se traduce en el paradigma del desarrollo territorial rural (DTR) que comenzó a permear la lógica de acción de los programas de desarrollo rural en Argentina hasta la actualidad. ${ }^{8}$

Ante este viraje, el concepto de agricultura familiar cobró un sentido más abarcativo que el de pequeño productor, minifundista o campesino, que hacía hincapié en las condiciones de pobreza o escasez (predial o de capital). Dado que el verdadero reto de la propuesta del DTR era poner en marcha procesos de transformación que incluyeran a los pobres, los excluidos y los micro y pequeños empresarios rurales, la categoría de agricultura familiar se constituyó en una noción más representativa de este abanico de actores.

8 Desde esta perspectiva, se recomienda una estrategia de desarrollo rural que contribuya a la cohesión y la inclusión social estimulando y facilitando la vinculación competitiva de los territorios rurales a mercados más dinámicos, la coordinación y las relaciones de cooperación entre los agentes económicos, sociales y gubernamentales y la inclusión de los sectores pobres y socialmente excluidos, así como también de las micro y pequeñas empresas agro-rurales en los procesos de transformación productiva y desarrollo institucional (Schejtman y Barsky, 2008) Esta definición amplia ha sido trabajada por un importante número de autores. Más recientemente, destacamos esta conceptualización para dar cuenta de políticas específicas o para discutir o problematizar la cuestión del desarrollo rural en particular. Véase Parafan et al. (2018) y Rodrigues Lopes (2015). 
Evidentemente, el rasgo familiar es el que ha otorgado consenso político a la categoría y el que ha posibilitado trascender las condiciones económicas de pobreza como indicadores exclusivos para su identificación. De este modo, entre fines del siglo pasado y principios del actual, la agricultura familiar fue creciendo en importancia como categoría teórica. Congrega políticamente a diversos actores rurales y se instala como problema de agenda de las políticas públicas de desarrollo rural en el país. Todo esto a partir del impulso generado desde los organismos internacionales y, fundamentalmente, de la recuperación de ciertas capacidades de los Estados nacionales en la región (Sabourin, Samper y Sotomayor, 2014; Salcedo, De la O y Guzmán, 2014). ${ }^{9}$

En este contexto, el agricultor familiar pasó de ser considerado estrictamente pobre o vulnerable a ser un agente socioeconómico con posibilidades de generar acciones que permitan su inclusión en el mercado y en las dinámicas ciudadanas. Es decir, se constituyó como un sujeto económico y político - o de derechos-, sobre el que se depositaron expectativas para la generación de procesos de desarrollo económico y social.

En Argentina, durante el período 2004-2014, se consolidó una visión compartida de la agricultura familiar como un sujeto activo de los procesos de desarrollo de los territorios rurales, a través, sobre todo, del fomento de las estrategias asociativas. Sin embargo, estas estrategias de acción tuvieron manifestaciones divergentes de acuerdo a las distintas agencias intervinientes en el área. Por un lado, se fomentó el asociativismo con fines productivos, comerciales y de agregado de valor, concibiendo la agricultura familiar como un sujeto económico. Por otro lado, se propició la consolidación de organizaciones con fines reivindicativos, destacando el carácter político de la agricultura familiar como un sujeto de derechos ciudadanos. Estas lógicas ${ }^{10}$ estuvieron en tensión durante todo el período y funcionaron como fuente de conflicto y discrepancias, pero también de consenso, en la búsqueda de cierta complementariedad de las acciones para propiciar procesos de desarrollo territorial que combinaran el crecimiento económico con la inclusión social de los sujetos (véase Nogueira, Urcola y Lattuada, 2017).

9 Cuando se hace referencia a la agricultura familiar, se destaca una temática en particular: la mano de obra familiar y su rol en la producción; en cambio, cuando utilizamos la categoría de pequeño productor o minifundista, se pone el acento en la escala de producción y en los problemas derivados de la limitada extensión de las explotaciones. La utilización de la categoría agricultura familiar amplía indudablemente la base de la población objetivo de un programa, incluyendo y dando visibilidad a un grupo de productores no tenidos en cuenta por términos como los de minifundista o campesino. En este concepto se incluyen también los denominados "productores familiares medianos o capitalizados" y aun población rural con actividades no agrarias (véase Nogueira y Urcola, 2013).

10 En trabajos anteriores identificamos estas lógicas en algunos programas del Fondo Internacional de Desarrollo Agrícola (FIDA) (radicados en la ex-UCAR) y en las acciones llevadas adelante por la ex Secretaría de Agricultura Familiar (SAF) (Nogueira y Urcola, 2015). 
Dicho todo esto, una de las novedades que ofrece la actualidad institucional del desarrollo rural en la Argentina es la resignificación del agricultor familiar como sujeto del desarrollo y su parcial desplazamiento discursivo por una figura muy presente en la propuesta oficial: la del emprendedor.

La coyuntura del período 2015-2019 muestra algunos virajes en esta concepción dual del sujeto en favor del perfil económico de la agricultura familiar que consideramos pertinente destacar y profundizar analíticamente. Tal como podremos observar, persiste un discurso vinculado a las organizaciones de la agricultura familiar con un perfil económico y productivo, pero ahora en tensión con acciones que promueven el desarrollo de las capacidades individuales de innovación y emprendedurismo de los sujetos.

\section{El emprendedurismo en las propuestas de desarrollo}

La figura del emprendedor no es ajena a la tradicional idea de desarrollo. De hecho, esta le otorga complejidad a la discusión, pues es la que primeramente introduce, desde la lógica de la modernización, la ideología de la igualdad de oportunidades, el self-made-man, el "obrero que se convierte en jefe", "el botones que se convierte en director", etcétera (Rist, 2002). En este marco, ¿qué es un emprendedor y por qué resulta importante introducirlo como un sujeto del desarrollo rural? En principio, un emprendedor no es estrictamente un empresario, sino un innovador que ofrece nuevas soluciones a viejos problemas.

La noción de emprendedor, que proviene del francés entrepreneur (pionero), ha tenido múltiples usos empíricos y teóricos en el marco de un campo de investigación en proceso de consolidación. Se la ha vinculado con la idea del "aventurero" que avanza hacia lo desconocido en contextos de incertidumbre. Esta idea es la que ha sido utilizada metafóricamente por diferentes corrientes de pensamiento, fundamentalmente económico, para identificar a aquellas personas que se inician en una empresa comercial o un proyecto con rasgos innovadores. Según Herrera Guerra y Montoya Restrepo (2013), el emprendedor nunca deja de ser innovador. Es quien tiene la idea o el concepto de un producto, servicio o actividad a realizar. En este sentido, se diferencia del empresario, cuyas cualidades se vinculan con la administración de un negocio.

Si bien pueden rastrearse antecedentes del uso del término en la literatura económica francesa de principios del siglo XVIII y en algunas referencias de los economistas clásicos británicos (como Adam Smith) vinculados al entrepreneurship (espíritu emprendedor) del business management, son las referencias a la "teoría de la dinámica económica" de Joseph Schumpeter y a la teoría de las oportunidades en mercados imperfectos de los economistas austríacos ${ }^{11}$ las que le darán forma al concepto.

11 A modo de ejemplo se pueden mencionar Von Mises, Hayek y Kirzner. 
Aunque Schumpeter no se propuso desarrollar una teoría sobre el emprendimiento, es él quien plantea la inestabilidad y la existencia de un "desequilibro dinámico" en los mercados, como consecuencia de las acciones de "destrucción creativa" que acompañan a los procesos de innovación llevados adelante por los empresarios. Esta perspectiva será luego retomada y complementada por los economistas austríacos, quienes van a asociar el término emprendedor con las acciones de especulación de aquellos que, a través del acceso diferencial a información y conocimientos, logran aprovechar y reconocer oportunidades en mercados signados por la incertidumbre (Formiche1la, 2004; Herrera Guerra y Montoya Restrepo, 2013).

Esta lógica del emprendedurismo impactará fuertemente en los planteos teóricos sobre el desarrollo local y rural a través de la propuesta de desarrollo humano o de las capacidades humanas, del economista y filósofo Amartya Sen. Según este autor, el desarrollo no consiste en el aumento de la oferta de bienes materiales y de su disponibilidad, sino en el crecimiento de las capacidades de los individuos para poder decidir y conducir su existencia (Lattuada, 2014). En este sentido, considera que las acciones de desarrollo de un territorio deben buscar que los individuos aumenten sus posibilidades de tener oportunidades de llevar adelante proyectos productivos, generando sus propios empleos e ingresos, mejorando su calidad de vida y generando valor agregado a todos los procesos económicos y sociales que hacen al funcionamiento de una comunidad (Duarte y Ruiz Tibana, 2009).

En las propuestas del desarrollo local (o endógeno) de mediados de los ochenta y su readaptación al enfoque del DTR, desde finales de la década del noventa y hasta la actualidad, ${ }^{12}$ encontramos acciones y programas que promueven el emprendedurismo y las empresas y organizaciones de la economía social como mecanismos de inserción sociolaboral en tiempos de crisis (Melián Navarro y Campos Climent, 2010). La intensidad y la escala del desempleo ${ }^{13}$ propiciaron la búsqueda de acciones gubernamentales y no gubernamentales que fomentasen la potencialidad y creatividad económica de

12 Las políticas europeas impulsoras del desarrollo local tienen su origen a principios de la década del ochenta con el Programa de Iniciativas Locales para la Creación de Empleo de la Organización para la Cooperación y el Desarrollo Económicos (OCDE) (1982-1985), continúan en la década del noventa con los programas LEDA (Local Economic Development Action) y se consolidan con las Iniciativas Comunitarias LEADER (Liasions Entre Actions de Développément de l'Economie Rural), LEADER Plus y PRODER (Programas Regionales de Desarrollo Rural) de fines de la década del noventa y principios de los 2000 (Melián Navarro y Campos Climent, 2010, p. 45). Tal como señalamos en el apartado anterior, estas propuestas vinculadas al desarrollo endógeno y local son las que van a influir en los planteos del DTR en nuestra región hacia mediados de la década de los noventa y principios del 2000 (Nogueira y Urcola, 2013).

13 Nos referimos a la particular tendencia al crecimiento económico sin generación de empleo durante la década neoliberal del noventa. 
grupos sociales vulnerables, como jóvenes, mujeres, trabajadores informales o minorías étnicas (Amin, 2008). Ante el fracaso de las políticas tradicionales de empleo, se comienzan a articular acciones enmarcadas dentro de los paradigmas del desarrollo local y territorial. Estos enfoques utilizaron como principal instrumento el impulso de las micro, pequeñas y medianas empresas de la economía social en sus múltiples formas jurídicas y asociativas. En estas perspectivas, las empresas y asociaciones de la economía social se presentaron, por un lado, como una forma de innovación económico-social y, por otro lado, como una forma de innovación político-gubernamental, en el marco de acciones que permitieran fomentar el autoempleo junto a emprendimientos y capacidades empresariales de base local (Amin, 2008; Melián Navarro y Campos Climent, 2010).

El emprendedurismo, dentro de la perspectiva de la economía social, ha intentado redireccionar el crecimiento económico hacia la inserción laboral y el desarrollo local. Se ha orientado al fomento de iniciativas que permitan la integración social y el crecimiento económico al mismo tiempo. No obstante, en buena parte de los programas y acciones para el desarrollo local o de los territorios rurales, las propuestas de inclusión social han quedado reducidas a propuestas de integración a mercados y los emprendimientos a planes de negocios. Por otro lado, los contextos de crisis en los que se han aplicado han tendido a transformar las iniciativas y motivaciones individuales en una coerción que se padece y atenta contra la vocación autonomista (del trabajo libre) que vincula el riesgo con una elección de los sujetos.

\section{El emprendedurismo como política del individuo o de la singularidad}

En la búsqueda de enfoques teóricos que nos permitan la interpretación/comprensión de este tipo de fenómenos discursivos y empíricos a nivel de las políticas públicas, resulta sugerente el planteo de Merklen (2013) acerca de las "políticas del individuo", propias de nuestro tiempo. El autor las concibe como aquellas cuyo foco está puesto en el individuo y no en las dinámicas sociales que, como tales, podrían resultar un obstáculo para la autonomía individual. Esto representa, en rigor, una reorientación de la relación Estado-sociedad. Uno podría pensar, advierte el autor, que las políticas que se orientan al individuo resultan exclusivamente políticas sociales, sin embargo, es posible considerar dispositivos de individuación en buena parte de los ámbitos sociales, entre los que podemos incluir los espacios rurales. Estos dispositivos obedecen a recursos cuyo fin es introducir un contenido específico a las acciones de los sujetos. En el caso de estas políticas, transmitir "responsabilidad" y activación" resultan los únicos contenidos viables, pues "cada persona debe definir el contenido de su experiencia y su proyecto de futuro" (Merklen, 2013, p. 74). Como puede observarse, estas nociones ape- 
lan a cierto laissez faire individual con evidentes efectos en la constitución de la sociedad. ${ }^{14}$

En cierto sentido, estas políticas construyen o, mejor dicho, reconstruyen, la concepción liberal moderna del individuo, desentendiéndose de cierta lógica de cuidados y bienestar de los paradigmas de carácter intervencionista. Por el contrario, el individuo será "responsable" de sí mismo y deberá mantenerse "activo". Se sostendrá en la "esfera privada" que el Estado solo tendrá que "proteger y respetar" (Merklen, 2013).

Estas políticas de individuación contemporáneas producen individuos que se perciben como objetos. ¿A qué individuos, entonces, se orientan estas políticas? A quienes han "perdido" o están "por perder". En palabras de Merklen (2013), a aquellos que, desestabilizados, se "cayeron del caballo" y necesitan ayuda para "volver al ruedo".

En esta misma línea argumental, Rosanvallon (2015) nos invita a contemplar las posibles ambigüedades de las actuales políticas de la singularidad. Las acciones que tienden a promover la realización del individuo, al mismo tiempo pueden volverse contra él. Su singularidad se ha convertido en un factor decisivo de la producción, en tanto su productividad reposa sobre su capacidad de movilizar recursos propios y de involucrarse de manera autónoma en una tarea. Pero este reconocimiento y llamado a la realización de las personas tiende a presentarse como un imperativo y a funcionar como una orden: "sean autónomos", "sean responsables", "tomen iniciativas" ("sean emprendedores"). De este modo, los procesos de individualización-emancipación se relacionan de manera confusa con procesos de individualizaciónfragilización (Rosanvallon, 2015, p. 328).

La ambigüedad en los usos del emprendedurismo en programas y políticas se vincula con las contradicciones y penetraciones empíricas y discursivas de lo que Ronsanvallon denomina "capitalismo de la singularidad" o "sociedad de la competencia generalizada". Con ambas ideas se refiere a la singularización de los procesos de trabajo (explotación de la fuerza de trabajo) y a la penetración material y simbólica de la economía de mercado en la

14 Esta lectura puede comprenderse también desde autores que ponen en duda las herramientas conceptuales de la sociología clásica para dar cuenta de los avances en los procesos de individuación. Conceptos tales como clase estarían en crisis: "progresivamente se impone la necesidad de reconocer la singularización creciente de las trayectorias personales" (Araujo y Martuccelli, 2010, p. 82). 
direccionalidad del orden social. ${ }^{15}$ El uso de estas nociones, cuyo desarrollo teórico quizá pone en duda su condición de conceptos, es un microindicador de los cambios radicales que acontecen en la sociedad actual y, asimismo, de los desafíos de las ciencias sociales para comprenderlos.

En síntesis, el emprendedurismo emerge como noción que intenta resaltar rasgos y atributos propios de los individuos y su desempeño, en el marco de transformaciones en el funcionamiento de la economía de mercado, la organización de la producción y las nuevas formas de inserción sociolaboral en contextos de crisis. No obstante, su inscripción en programas y políticas de las últimas décadas lo integran discursivamente en el segmento de las "políticas del individuo" o de la "singularidad" a las que aluden Merklen (2013) y Rosanvallon (2015).

Como veremos a continuación, las acciones que se sostienen discursivamente alrededor del desarrollo rural y la agricultura establecen una disputa de sentido en torno al rol de los sujetos de la política pública (en este caso, el agricultor familiar) y sus formas de relacionarse con el Estado. Esta impronta tiende a romper con las lógicas preponderantes en las acciones precedentes.

\section{De agricultor familiar a emprendedor innovador}

Para comprender el contexto de penetración discursiva del emprendedurismo en el marco de las acciones del desarrollo rural actual, debemos reseñar muy brevemente algunos cambios significativos en el interior del Estado argentino. El primer movimiento significativo fue una nueva modificación en la Ley de Ministerios, por la cual el Ministerio de Agricultura, Ganadería y Pesca (creado en 2009) se convirtió en Ministerio de Agroindustria, en diciembre de 2015. Luego, en marzo de 2018, se concretó una profunda reforma de su estructura. Se redujeron numerosas áreas con el objetivo de "desburocratizar" y achicar la planta política; se eliminaron más de ochenta direcciones y coordinaciones y se despidieron alrededor de novecientos trabajadores en todo el país, en su mayoría personal técnico de la Secretaría de Agricultura Familiar (SAF). Finalmente, en septiembre de 2018, en el marco de una crisis cambiaria y con la intención de reducir el déficit fiscal, acorde con las normativas del Fondo Monetario Internacional (FMI), el Poder Ejecutivo promulgó el Decreto 801/2018, que estableció la reducción del número de ministerios

15 En el capitalismo contemporáneo, la movilización del obrero-masa fordista dio paso a una valorización de las capacidades individuales de creación y las cualidades de reactividad suplantaron el sentido de disciplina, generando un efecto de singularización del trabajo. A su vez, sostiene la idea de una sociedad de competencia generalizada como una forma radicalizada de la sociedad de mercado y del orden de mercado basado en la noción de igualdad de oportunidades. Dicho orden promueve la competencia como forma genérica del lazo social, sacraliza la figura del consumidor como medida del interés general y sostiene una filosofía y antropología del riesgo como condición ontológica del hombre (Rosanvallon, 2015, p. 290). 
y la degradación del Ministerio de Agroindustria al rango de Secretaría, pasando a depender del nuevo Ministerio de Producción y Trabajo.

En lo que refiere concretamente al área de desarrollo rural y de agricultura familiar, dentro de la cartera de Agroindustria, entre 2016 y 2018, la nueva Secretaría de Agricultura Familiar, Coordinación y Desarrollo Territorial (SAFCyDT) ${ }^{16}$ se ha caracterizado más por la paralización, evaluación y redefinición de las acciones realizadas por la gestión anterior, que por iniciativas propias de envergadura dirigidas específicamente hacia los agricultores familiares. Por ello, muchas de las acciones que podemos reseñar tienen un carácter más discursivo y hasta propagandístico que de impacto, en términos de acciones que se traduzcan en partidas presupuestarias e instrumentos de intervención de relevancia. ${ }^{17}$

La gestión actual de esta secretaría ha redefinido al sujeto de sus intervenciones, estableciendo una clara distinción entre los sujetos de asistencia social (los "pobres" o vulnerables) y los sujetos de crédito o de acceso a incentivos económicos para emprendimientos rurales o para la comercialización de productos agropecuarios (los "productores viables").

La eliminación del monotributo social agropecuario (MSA) se destaca entre las acciones que han transformado el escenario institucional del desarrollo rural y la agricultura familiar en Argentina. ${ }^{18}$ Esta medida obliga a los sujetos que no pueden sostener su actividad en forma autónoma a inscribirse en el monotributo social (MS), administrado por el Ministerio de Desarrollo Social.

16 La nueva secretaría fusiona tres secretarías de la gestión anterior: la de Agricultura Familiar, la de Coordinación Política y la de Desarrollo Territorial.

17 En el presupuesto aprobado por el Congreso Nacional en 2016 se destacan dos iniciativas centrales para el sector: 1) el Plan Belgrano, enfocado prioritariamente en la creación de infraestructura asociada con las necesidades productivas y agroexportadoras, sin desconocer en un segundo nivel las consideraciones sobre acciones para combatir la pobreza, los déficits habitacionales y los incentivos laborales, y 2) el Fondo Fiduciario Nacional de Agroindustria (FONDAGRO), en el cual la agricultura familiar aparece mencionada residualmente y sin líneas específicas de acción (véase Nogueira et al., 2017). Ambas iniciativas representan acciones de envergadura, aunque no específicas para los sectores comprendidos por la agricultura familiar, y se encuentran hoy paralizadas por el ajuste presupuestario que se puso en marcha tras el inicio de las negociaciones del gobierno nacional con el FMI. A estas se pueden sumar las acciones de una serie de programas con financiamiento externo concentrados en la actual Dirección General de Programa y Proyectos Sectoriales y Especiales (DIPROSE, ex-UCAR), que ya estaban en ejecución desde la gestión anterior (PRODERI, PROCANOR, PRODESPA, PRODAF, PISEAR, PRODECCA, PROSAP) y que articulan sus acciones con la SAFCyDT.

18 A partir de un convenio celebrado entre el MAGyP y el Ministerio de Desarrollo Social de la Nación en 2009, los productores de la agricultura familiar podían acceder al MSA sin costo alguno. Esta herramienta facilitaba su incorporación a la economía formal y posibilitaba el acceso al sistema integrado de seguridad social (jubilación) y la prestación de obra social para la salud del productor y su grupo familiar primario. En este sentido, el MSA podía ser considerado como un instrumento de inclusión que otorgaba "ciudadanía tributaria" y se presentaba como un "generador de derechos" que no excluía otro tipo de prestaciones como la asignación universal por hijo (Nogueira et al., 2017, p. 40). 
En relación con los incentivos económicos para emprendedores rurales o para la comercialización, pueden destacarse las siguientes acciones: a) la presentación del sello Producido por la Agricultura Familiar (Res. 330-E/2017), un isologotipo que, además de identificar y reivindicar los atributos de la producción de los agricultores familiares, "certifica la trazabilidad, inocuidad y el origen social de los productos que llegan a las góndolas" (Ministerio de Ganadería, Agricultura y Pesca Argentina, 2017); b) la inclusión de productos de la agricultura familiar en el Código Alimentario Argentino (CAA), que simplifica los requisitos para la habilitación de los establecimientos de pequeña y mediana escala que elaboran y comercializan alimentos caseros, artesanales y tradicionales; ${ }^{19}$ c) el Plan Nacional de Inclusión Digital de la Agricultura Familiar, que ofrece capacitaciones para el uso de herramientas tecnológicas digitales que permitan "potenciar las oportunidades de comercialización, desarrollando habilidades para el acceso a los mercados de estos actores" (Ministerio de Ganadería, Agricultura y Pesca Argentina, 2019); y d) la implementación del programa AgroEmprende "Innovando en el Territorio", que se propone generar "el entorno propicio para la detección de oportunidades, el acompañamiento en la formulación de negocios y el acceso a recursos para la puesta en marcha o fortalecimiento de emprendimientos agroindustriales para los jóvenes rurales" (Ministerio de Agroindustria de la Nación Argentina, 2017b), y el Programa Argentina Emprende, que es una iniciativa de la Secretaría de Agroindustria, la SAFyDT y el Banco de la Nación Argentina para implementar un mecanismo operativo que facilite el acceso al crédito a emprendedores, microempresas agroproductivas de la agricultura familiar y de las economías regionales. ${ }^{20}$ Con esta iniciativa, afirma el secretario de Agroindustria, se "fortalecerá el desarrollo de los diversos sectores agroproductivos vinculados a la agricultura familiar, impulsando su formalización, modernización, reconversión, complementación, como también la diversificación de las economías regionales" (Ministerio de Ganadería, Agricultura y Pesca Argentina, 2018a).

\section{El fomento de la cultura emprendedora a través de los programas de desarrollo rural}

Los primeros antecedentes de esta línea de intervención destinada a promover la cultura emprendedora de los sujetos del agro podemos encontrarlos en un proyecto piloto destinado a los jóvenes rurales, en el marco de los programas de desarrollo rural con financiamiento externo entre los años 2005 y

19 Contempla encurtidos, mermeladas, panificados, quesos, dulce de leche y yerba mate (INTA, 2019).

20 Debe destacarse la sanción de una norma nacional que enmarca y habilita estos procesos asociados al capital emprendedor en términos generales. Nos referimos a la Ley 27.349, de Apoyo al Capital Emprendedor, que data del año 2017. 
2009. ${ }^{21}$ En este período se llevó adelante el Proyecto Piloto Jóvenes Emprendedores Rurales (PJER), como parte del Programa de Servicios Agrícolas Provinciales (PROSAP) de la ex Secretaría de Agricultura, Ganadería, Pesca y Alimentación y que luego sería incorporado a la Secretaría de Desarrollo Rural y Agricultura Familiar (en el marco del Ministerio de Agricultura, Ganadería y Pesca de 2009). El proyecto tenía como objeto "fortalecer el espíritu emprendedor de los jóvenes rurales mediante la promoción de las capacidades individuales para la creación de nuevos emprendimientos" (PROSAP, 2009, p. 3). Se planteó como estrategia incrementar la cantidad de jóvenes ${ }^{22}$ que inicien sus nuevos negocios. La letra del proyecto es clara en la definición del objetivo: "promoviendo la diversificación productiva y la actividad innovadora a través de la creación de nuevos negocios que posibiliten la permanencia de los jóvenes en sus localidades, atenuando la migración campo-ciudad y fortaleciendo el desarrollo sostenible del sector agropecuario argentino" (PROSAP, 2009, p. 3).

Este proyecto piloto funcionó como una herramienta más dentro de una batería de acciones llevadas adelante en el marco de otros programas. ${ }^{23} \mathrm{Se}$ focalizaba en los jóvenes rurales a través de acciones que integraban lo productivo, lo educativo y la inserción laboral dentro de la perspectiva de la cultura emprendedora, a través de la creación de Centros de Desarrollo Emprendedor. Según el informe final del proyecto, en el período 2006-2009 se crearon 220 unidades de producción, se fortalecieron 568 emprendimientos y se brindó asistencia a 1.200 jóvenes para el desarrollo de sus proyectos en todo el país. Del total, 451 planes de negocios fueron efectivamente financiados (37\%) a partir de su evaluación técnica de sustentabilidad (PROSAP, 2009). ${ }^{24}$

A su vez, en el marco de este proyecto se fomentó una línea de formación en liderazgo y gestión de las organizaciones sociales. El propósito era apoyar la demanda de organizaciones agrarias ${ }^{25}$ y consolidar el campo asociativo y reivindicativo de la agricultura familiar en el marco de los Foros Nacionales de la Agricultura Familiar (FoNAF), por aquel entonces aún en construcción.

Este proyecto piloto fue marginal en un contexto general de expansión y jerarquización institucional de las acciones para el desarrollo de los territorios rurales y la agricultura familiar (Lattuada, Nogueira y Urcola, 2015). El Programa AgroEmprende, en cambio, surge como una de las acciones concretas de la actual gestión, que da relevancia analítica a nuestra hipótesis

21 Véase PROSAP, 2009, y Fondo Internacional de Desarrollo Agrícola (FIDA), 2010.

22 Considerando como tales a los comprendidos entre 15 y 29 años.

23 Por ejemplo, el PRODERNEA y el PRODERNOA del FIDA.

24 De ellos, 294 fueron financiados por el programa y 157 a través de terceros.

25 En especial de la Secretaría de Juventud de la Federación Agraria Argentina (FAA). 
sobre el emprendedurismo como lógica hegemónica que unifica el carácter de las intervenciones de la nueva SAFCyDT.

Según expresa su página oficial, AgroEmprende se promueve como un canal para "vincular el espíritu emprendedor de todos aquellos jóvenes que formen parte del programa, con aquellas empresas que desarrollan sus actividades en el sector y puedan participar apoyando aquellos proyectos que sean de su interés" (Ministerio de Ganadería, Agricultura y Pesca Argentina, $2018 b$ ). A través de esta alianza público-privada se busca potenciar el arraigo y fortalecer el desarrollo sostenible con propuestas productivas, agroindustriales y de servicios e impulsar la identificación de proyectos para la juventud rural como una herramienta para favorecer la creación de empleo y el acceso a oportunidades.

Para cumplir este objetivo se realizan convocatorias, encuentros federales, incubación de proyectos y se brinda asesoramiento. En su ejecución, el programa realiza una convocatoria a proyectos productivos. Luego, articulando con otros organismos públicos y privados, se realiza la implementación y evaluación de los proyectos seleccionados. Se conforma una red de instituciones de promoción del emprendedurismo y se brinda acceso a los recursos para la puesta en marcha o el fortalecimiento de los emprendimientos agroindustriales.

En el mes de septiembre de 2016 se realizó la experiencia piloto del programa en la provincia de Catamarca, a través de la convocatoria de proyectos productivos para jóvenes de entre 18 y 40 años. ${ }^{26}$ Los inscriptos superaron las 1.100 personas y se brindaron cinco capacitaciones a lo largo de dos meses. Como resultado se presentaron más de 300 proyectos, de los cuales resultaron seleccionados y financiados 45 (15\%). En esta convocatoria participaron los ministerios de Agroindustria y de Producción de la Nación, el Ministerio de la Producción de la Provincia de Catamarca, la Asociación de Emprendedores de Argentina (ASEA) y la Universidad Nacional de Catamarca (UNCA). Entre 2017 y 2018 esta experiencia se replicó en las provincias de Buenos Aires, Jujuy, Chubut y Formosa. Para el año 2019 se pretendió abarcar un total de 16 provincias, capacitando a 8.000 jóvenes. El propósito fue seleccionar alrededor de 4.000 proyectos, de los cuales fueron financiados $800(20 \%)$.

\section{Análisis de los programas de fomento de la cultura emprendedora}

¿Qué es lo que tienen en común las experiencias piloto PJER y AgroEmprende? ¿Qué las diferencia de otros programas de desarrollo rural? En principio,

26 Nótese que se modifica el rango etario respecto del PJER. Se eliminó a los adolescentes de entre 15 y 17 años y se amplió la edad hasta los 40 años. Esta cuestión permite una mayor inclusión de personas en edad económicamente activa que supera la tradicional referencia a la juventud (hasta 30 años), vinculada más con atributos simbólicos (innovación, adaptación al cambio, etcétera) que empíricos. 
su focalización en los jóvenes y su apuesta a la formación para la elaboración de proyectos sometidos a evaluación. Al igual que en la mayoría de las acciones vinculadas al emprendedurismo en diferentes ámbitos, los jóvenes son los destinatarios de estas iniciativas. Intervenciones de este tipo persiguen un cambio cultural como base de un proceso de crecimiento y desarrollo económico, vinculando inclusión con competencia de mercado. Desde esta perspectiva, hay una estrecha relación entre innovación, sociedad del conocimiento y mentalidad empresarial, que se motoriza a través de cursos y seminarios en los cuales se transmiten las capacidades, habilidades y actitudes que los jóvenes deberán incorporar (o descubrir que "ya están dentro de ellos") y traducir en una propuesta o iniciativa (proyecto o plan de negocios) para insertarse laboralmente e integrarse a la sociedad de mercado.

La promoción del emprendedurismo implica el diseño de acciones que combinen lo educativo con lo laboral, circunscribiendo la formación educativa a las demandas del mercado. Por ello, Escuelagro es la otra iniciativa que se articula con el programa AgroEmprende (véase Infocampo, 2016). En el marco de las Jornadas Innovar y Repensar la Educación Agraria, el secretario de la SAFCyDT señaló que "a través de Escuelagro proponemos actualizar conocimientos y conectar el mundo productivo con el educativo", y agregó que "el emprendedurismo es fundamental para armar empresas de base local a través de las cuales se va a generar más trabajo que es lo que falta para el desarrollo profundo del interior del país" (Ministerio de Agroindustria de la Nación Argentina, 2017a).

Ahora bien, el punto central y tal vez el que lo diferencia en forma más significativa de los otros programas de desarrollo rural es que su implementación contiene un concurso en el que se inscriben muchos participantes y solo algunos resultan ganadores (con premios y menciones), en función de una evaluación y selección de proyectos, de acuerdo a criterios preestablecidos. Desde sus primeras experiencias, la mayoría de los programas de desarrollo rural han funcionado con algún criterio de focalización. No obstante, los criterios de focalización de estos programas han tendido a plantearse entre sus objetivos un carácter lo más universal posible, en el marco de acciones de extensión hacia las comunidades rurales (apoyo técnico, financiamiento, etcétera), como camino para la generación de procesos de desarrollo y crecimiento económico de los territorios. Esto se observa en la evaluación final de algunos programas en los que se pretende mostrar - en ocasiones de manera exagerada - la cantidad de personas y familias beneficiarias. En cambio, en la modalidad de programas de promoción del emprendedurismo a través de concursos, la difusión de sus bases y condiciones y el legítimo proceso de selección de los participantes son los pilares fundamentales. Los proyectos seleccionados para estos programas se suelen presentar en actos oficiales de 
entrega de premios y estímulos financieros. ${ }^{27}$

Este formato promueve la idea de una sociedad que ofrece igualdad de oportunidades para la competencia. El programa funciona como plataforma que, a través de la competencia regulada, garantiza la posibilidad de participación de todos los interesados, quienes serán premiados en función de sus méritos e iniciativas individuales (o de equipo), y quienes no lo logren contarán con la promesa y el incentivo para seguir participando.

En la era del capitalismo de la singularidad, siguiendo a Rosanvallon (2015), hay dos maneras de justificar y articular el principio de igualdad con el de las diferencias sociales: el azar o el mérito. El azar es estructuralmente igualador, ya que nadie puede apropiárselo y, psicológicamente, tiene la virtud de permitir atribuir a la mala suerte un fracaso o posición desfavorable, atenuando la responsabilidad. En cambio, el mérito incluye variables de naturaleza (talentos) y de comportamiento (virtud) que relacionan capacidad individual con esfuerzo en el marco de algún tipo de competencia regulada. El mérito funciona como una "ficción necesaria" que tiene por objeto revelar capacidades naturales y cualidades mentales, permitiendo a los individuos dar sentido a su existencia (el jugador es el único artesano de su destino) y conciliar el principio filosófico de igualdad con el hecho social de las desigualdades de posiciones. No obstante, el autor advierte que la referencia simplista al mérito oculta el hecho de que la apreciación de las "capacidades" en competencia depende de las representaciones que uno se haga de una buena sociedad y de la ciencia del hombre a la cual se haga referencia implícitamente (Rosanvallon, 2015, p. 281).

En el marco de estos programas por concurso, el Estado cumple una función legitimadora (efecto simbólico) y performativa a través de la construcción de la "ficción" que permite la selección de los talentos y cualidades que se pretenden motorizar. En este sentido, la idea de igualdad de oportunidades es inseparable del ethos meritocrático que, según Rosanvallon (2015), da lugar a una nueva "aristocracia del talento" que tiende a justificar las desigualdades y desestimar las discusiones en torno al acceso a derechos sociales universales (nivel de recursos que deben ser garantizados por la sociedad para todos).

De este modo, el sujeto de las políticas y los programas de desarrollo rural, que anteriormente se intentaba englobar en la noción amplia de agricultura familiar y al que se asistía a través de acciones que combinaban instrumentos de acceso a derechos con programas de acceso a financiamiento y asesoramiento técnico-productivo, se encuentra en tensión. Los

27 En julio de 2017 se entregaron los premios AgroEmprende Buenos Aires. "En total participaron 378 propuestas, de las cuales fueron premiadas 68 , focalizadas en potenciar la innovación entre jóvenes de 18 a 40 años, y apuntando a la creación de nuevas empresas locales, que contribuyan al desarrollo económico de la provincia. Se entregaron \$ 5 millones en premios" (Ministerio de Agroindustria de la Nación Argentina, 2017a). 
cambios institucionales que acontecieron fundamentalmente en el interior de la ex-SAF parecen transformar paulatinamente las herramientas de acceso a derechos e inclusión ciudadana (como el MSA) en instrumentos de asistencia a la pobreza. Al mismo tiempo, existe cierta ambigüedad e indefinición en el tipo de productor al que se orientan las acciones en la órbita institucional del desarrollo rural y la agricultura familiar. La referencia a los "no tan jóvenes" emprendedores rurales (de hasta 40 años) que promueven los nuevos programas y las líneas de asistencia a la comercialización (sello de agricultura familia, inclusión digital y AgroEmprende), llevados adelante por la SAFCyDT, se caracterizan por plantear una difusa o escasa distinción entre los pequeños productores de base familiar y las pequeñas o medianas empresas de base local.

\section{Reflexiones finales}

Desde diciembre de 2015, observamos cambios radicales en el interior del Estado en Argentina, que ocurren en paralelo con procesos similares en otros países del continente latinoamericano (Sabourin, 2018). Estos cambios no se asientan solo en la reducción del aparato estatal y los espacios de gestión de algunas áreas, sino también, y en especial, en la orientación de las políticas públicas y la formación de los recursos humanos que participan de esos ámbitos. El desarrollo rural y la agricultura familiar conforman una de estas áreas en "transformación".

En este artículo mostramos que, a pesar de la incertidumbre del contexto, es posible visualizar un conjunto de cuestiones que marcan con claridad una tendencia prevaleciente. Al inicio presentamos dos visiones sobre las acciones vinculadas con el desarrollo rural y la agricultura familiar, que se consolidan fundamentalmente entre 2004 y 2014. Entre dichas posiciones, destacamos cierta orientación a la construcción de ciudadanía social en sectores vinculados con la agricultura familiar llevados adelante por la ex-SAF, como algo novedoso respecto de las acciones de asistencia técnica y productiva de larga tradición en los programas de desarrollo rural. Por el contrario, a partir de 2015 observamos cómo, en forma sorprendentemente rápida, tal visión casi desaparece de los espacios de gestión, dando lugar a otras lógicas que creemos presentan una propensión hegemónica, de acuerdo a lo que se manifiesta en algunos de los instrumentos, programas y proyectos que hemos reseñado.

Entre 2015 y 2019, se desarrolló un proceso de desmantelamiento de las estructuras de gestión estatal vinculadas con la agricultura familiar (desfinanciamiento, despidos de personal, paralización de programas e instrumentos de asistencia). No obstante, en este artículo hemos querido destacar cómo estos cambios están siendo acompañados, en el plano discursivo, por un sistemático desplazamiento y desdibujamiento de la agricultura familiar como sujeto de las políticas públicas de desarrollo rural. 
Las herramientas de intervención elaboradas por la actual gestión de la SAFCyDT, desde nuestra perspectiva, tienden a dar centralidad a la noción de emprendedurismo. Los programas por concurso, sobre los que nos detuvimos a lo largo de este artículo, son, hasta el momento, la mayor expresión de la tendencia a otorgar al emprendedor en sí mismo un carácter transformador de su propio contexto. Si bien ya existían, se han extendido y propagado en este nuevo contexto y marcan, en rigor, la orientación de las acciones de desarrollo rural vinculadas con innovación, desarrollo personal, mérito y conocimiento.

En este sentido, se modifica la referencia al agricultor familiar antes concebido como un sujeto económico, pero también como un sujeto político $\mathrm{y}$, esencialmente, de derechos. Ese agricultor familiar resulta ahora un emprendedor. Como tal, la responsabilidad de éxito descansa exclusivamente en sus aptitudes y su "olfato" frente a la actividad que escoja, siendo el Estado - en última instancia - el que garantiza la igualdad de oportunidades. Lo anterior, en definitiva, redefine el rol de la política en cuanto a su intervención e instaura, como en otras oportunidades, una lógica del laissez faire por parte del Estado, limitando su intervención.

En realidad, emprender tiene un carácter performativo que se vincula con los rasgos centrales del management, tendencia que pareciera reproducirse en las diversas áreas del Estado nacional. Si bien esto no siempre se encuentra expuesto de modo explícito, nos arriesgamos a afirmar que el perfil del agricultor familiar que interesa sostener es el del emprendedor rural, asociado a los modelos empresariales de los que antes pretendía diferenciarse. Tengamos en cuenta que la noción de agricultura familiar que primó durante el período 2004-2014 tuvo amplias y múltiples definiciones y acepciones (pequeña, mediana, capitalizada, de subsistencia, etcétera), pero esencialmente se presentó como una forma de nombrar y aglutinar a los sujetos y formas de hacer agricultura y habitar los territorios rurales que se diferenciaban del modelo de agricultura empresarial.

La exaltación por el hacer individual y su carácter emprendedor tiñe las acciones de desarrollo rural dirigidas a la agricultura familiar. Siendo críticos de los procesos aquí analizados, la pregunta respecto de quiénes son parte del universo que comprende a los sujetos de las políticas para el desarrollo de los territorios rurales hoy se responde de acuerdo a criterios que se relacionan con el carácter individual de los sujetos y sus capacidades de innovación para la creación de oportunidades de integración social y económica. Será cuestión de ver cómo permanecen estos atributos estructuralmente, más allá de las acciones de gobierno que hoy nos toca atender. 


\section{Referencias bibliográficas}

Amin, A. (2008). Una perspectiva institucionalista sobre el desarrollo económico regional. En: V. R. Fernández, A. Amin y J. I. Vigil. Repensando el desarrollo regional. Contribuciones globales para una estrategia latinoamericana. Buenos Aires: Miño y Dávila, pp. 101-120.

Araujo, K. y D. Martuccelli (2010). La individuación y el trabajo de los individuos. Educação e Pesquisa, 36, 77-91.

Bertoni, L. y S. Soverna (2015). La transición de las políticas públicas para la agricultura familiar en Argentina: de los programas a la Ley. Ponencia presentada en IX Jornadas Interdisciplinarias de Estudios Agrarios y Agroindustriales, Buenos Aires, noviembre.

Chiriboga, M. (1996). Desafios de la pequeña agricultura familiar frente a la globalización. Ponencia presentada en Congreso de la Asociación Latinoamericana de Economistas Agrícolas (ALACEA), San José de Costa Rica, septiembre.

Formichella, M. (2004). El concepto de emprendimiento y su relación con la educación, el empleo y el desarrollo local. Tres Arroyos: INTA.

Duarte, T. y M. Ruiz Tibana (2009). Emprendimiento, una opción para el desarrollo. Scientia Et Technica, XV(43), 326-331.

Fondo Internacional de Desarrollo Agrícola, FIDA (2010). Jóvenes emprendedores rurales. Una aproximación a la evolución y estado actual de los servicios de apoyo a América Latina. Santiago de Chile: PROCASUR-FIDA.

Herrera Guerra, C. E. y L. A. Montoya Restrepo (2013). El emprendedor: una aproximación a su definición y caracterización. Punto de Vista, IV(7), 7-30.

Infobae (2018). Hubo incidentes frente al Ministerio de Agroindustria. Infobae, 31 de agosto. Disponible en: <https:/www.infobae.com/politica/2018/08/31/ tension-frente-al-ministerio-de-agroindustria/> [acceso 20/09/2018].

Infocampo (2016). Se creó Escuelagro, un programa para vincularla educación rural y la producción agroindustrial. Infocampo, 2 de noviembre. Disponible en: <http://www.infocampo.com.ar/se-creo-escuelagro-un-programapara-vincular-la-educacion-rural-y-la-produccion-agroindustrial/> [acceso 14/09/2018]. 
Instituto Nacional de Tecnología Agropecuaria (INTA) (2019) El Código Alimentario incorpora productos de la agricultura familiar. INTA Informa, 8 de enero. Disponible en: $<$ http://intainforma.inta.gov.ar/?p=46291 $>$ [acceso 04/02/2019].

Lattuada, M. (2014). Políticas de desarrollo rural en la Argentina. Conceptos, contexto y Transformaciones. Temas y Debates, 18(27), 13-47.

Lattuada, M.; M. E. Nogueira y M. Urcola (2015). Tres décadas de desarrollo rural en la Argentina. Continuidades y rupturas de intervenciones públicas en contextos cambiantes (1984-2014). Buenos Aires: Teseo.

Maletta, H. (2011). Tendencias y perspectivas de la agricultura familiar en América Latina. Santiago de Chile: RIMISP.

Manzanal, M. (2006). Regiones, territorios e institucionalidad del desarrollo rural. En: M. Manzanal, G. Neiman y M. Lattuada (comps.). Desarrollo rural: organizaciones, instituciones y territorios. Buenos Aires: Ciccus, pp.21-50.

Melián Navarro, A. y V. Campos Climent (2010). Emprendedurismo y economía social como mecanismos de inserción sociolaboral en tiempos de crisis. $R E-$ VESCO, Monográfico: La respuesta de la Economía Social ante una crisis global, 100, 43-67.

Merklen, D. (2013). Las dinámicas contemporáneas de la individuación. En: R. Castel, G. Kessler, D. Merklen y N. Murard. Individuación, precariedad, inseguridad ¿Desinstitucionalización del presente? Buenos Aires: Paidós, pp. 45-86.

Ministerio de Agroindustria de la Nación Argentina (2017a). Entregaron los premios Agroemprende Buenos Aires. Ministerio de Agroindustria de la $\mathrm{Na}$ ción Argentina, 24 de julio. Disponible en: <http://www.minagri.gob.ar/sitio/areas/agroemprende/?accion $=$ noticia\&id_info $=170724190443>$ [acceso 14/09/2018].

Ministerio de Agroindustria de la Nación Argentina (2017b). Resolución 150E/2017. Buenos Aires, 14 de junio. Disponible en: $<$ http://servicios.infoleg. gob.ar/infolegInternet/anexos/275000-279999/275909/norma.htm $>$ [acceso $12 / 09 / 2018]$.

Ministerio de Agroindustria de la Nación Argentina (2017c). Se realizó con éxito la Jornada Innovar y Repensar la Educación Agraria. Ministerio de Agroindustria de la Nación Argentina, 12 de diciembre. Disponible en: <https:// 
www.agroindustria.gob.ar/sitio/areas/escuelagro/?accion=noticia\&id info $=171212101844>$ [acceso 19/09/2018].

Ministerio de Ganadería, Agricultura y Pesca Argentina (2017). Se reglamentó el uso del Sello Producido por la Agricultura Familiar. Ministerio de Ganadería, Agricultura y Pesca Argentina, 6 de noviembre. Disponible en: $<$ https://www.agroindustria.gob.ar/sitio/areas/prensa/?accion=noticia\&id_ info $=171106092948>$ [acceso 19/09/2018].

Ministerio de Ganadería, Agricultura y Pesca Argentina (2018a). Facilitarán el acceso al crédito a microempresas de las economías regionales y de la agricultura familiar. Página oficial del gobierno argentino, 26 de diciembre Disponible en: <https://www.argentina.gob.ar/noticias/facilitaran-el-acceso-al-credito-microempresas-de-las-economias-regionales-y-de-la $>$ [acceso $04 / 02 / 2019$ ].

Ministerio de Ganadería, Agricultura y Pesca Argentina (2018b). Agroemprende. Ministerio de Ganadería, Agricultura y Pesca Argentina. Disponible en: $<$ https://www.agroindustria.gob.ar/sitio/areas/agroemprende/institucional/> [acceso 14/09/2018].

Ministerio de Ganadería, Agricultura y Pesca Argentina (2019). Presentaron el Plan Nacional de Inclusión Digital para la Agricultura Familiar. Ministerio de Ganadería, Agricultura y Pesca Argentina, 12 de abril. Disponible en: <http://www.minagri.gob.ar/sitio/areas/prensa/index. php?accion=noticia\&id_info=180412151433 $>$ [acceso 12/09/2018].

Nogueira, M. E. y M. Urcola (2013). La agricultura familiar en las políticas de desarrollo rural, ¿hacia una nueva agenda pública? La experiencia reciente en Argentina (1990-2011). Revista Interdisciplinaria de Estudios Agrarios, 39, 5-38.

Nogueira, M. E. y M. Urcola (2015). La agricultura familiar en el marco de los programas de desarrollo rural del FIDA en el norte argentino. Revista AGER, 19, 7-44.

Nogueira, M. E.; M. Urcola y M. Lattuada (2017). La gestión estatal del desarrollo rural y la agricultura familiar en Argentina: estilos de gestión y análisis de coyuntura 2004-2014 y 2015-2017. Revista Latinoamericana de Estudios Rurales (ReLaER), 2(4), 23-59. 
Página 12 (2018). La tijera les llegó a los pequeños productores. Página 12, 29 de junio. Disponible en: $<$ https://www.pagina12.com.ar/125113-la-tijerales-llego-a-los-pequenos-productores $>$ [acceso 17/09/2018].

Parafan, M.; M. Balestro; D. Sayago y E. Sabourin (2018). Difusión regional de políticas de desarrollo territorial rural. Mundos plurales, 2, 51-72.

Poder Legislativo-Argentina (2017). Ley 27.349 de Apoyo al Capital Emprendedor. Buenos Aires, 29 de marzo. Disponible en: $<\mathrm{http}$ :/servicios.infoleg. gob.ar/infolegInternet/anexos/270000-274999/273567/texact.htm> [acceso 26/04/2019].

Programa de Servicios Agrícolas Provinciales, PROSAP (2009). Proyecto piloto jóvenes emprendedores rurales. "Promoción de la cultura emprendedora". Informe final del proyecto. Buenos Aires: PROSAP, Ministerio de Agricultura, Ganadería y Pesaca (MAGyP), Banco Mundial (BM).

Rist, G. (2002). El desarrollo: historia de una creencia occidental. Madrid: Los Libros de la Catarata.

Rodrigues Lopes, G. (2015). Desmontando el desarrollo territorial rural (DTR) en América Latina. Tabula Rasa, 23, 181-202.

Rofi, D. (2018). Agroindustria comunicó el fin del Monotributo Social Agropecuario. La Nación, 29 de junio. Disponible en: <https://www.lanacion.com. ar/2148534-agroindustria-comunico-el-fin-del-monotributo-social-agropecuario $>$ [acceso 17/09/2018].

Rosanvallon, P. (2015). La sociedad de iguales. Buenos Aires: Manantial.

Salcedo, S.; A. P. de la O y L. Guzmán (2014). El concepto de agricultura familiar en América Latina y el Caribe. En: S. Salcedo y L. Guszmán (edits.). Agricultura familiar en América Latina y el Caribe: recomendaciones de política, Santiago de Chile: FAO, pp. 17-29.

Sabourin, E.; M. Samper y O. Sotomayor (coords.) (2014). Políticas públicas y agriculturas familiares en América Latina y el Caribe. Balance, desafios y perspectivas. Santiago de Chile: CEPAL-IICA.

Sabourin, E. (2018). Erosão, crise e desmonte de políticas para a agricultura familiar e agroecologia na America Latina. Trabajo presentado en Seminario Políticas Públicas para o Meio Rural Brasileiro no Período Recente: Mudanças, Continuidades e Rupturas, Río de Janeiro, diciembre. 
Schejtman, A. y J. Berdegué (2004). Desarrollo territorial rural. Temas y Debates Rurales, 1, 1-54.

Schejtman, A. (2008). Alcances de la Agricultura familiar en América Latina. Santiago de Chile: RIMISP.

Schejtman, A. y O. Barsky (comps.) (2008). El desarrollo rural en la argentina. Un enfoque territorial. Buenos Aires: Siglo XXI.

Toril, J.y J.Valenciano(2011). Revisando el emprendedurismo. Boletín Económico del ICE, (3021) [en línea]. Disponible en: <http://www.revistasice.com/CachePDF/BICE_3021_53-62_E596526B3F4F228C856BBD7657107DD8. pdf $>$ [acceso 18/03/2018].

Urcola, M. (2016). Caracterización de la agricultura familiar a partir de un programa de desarrollo rural: el caso de los destinatarios del PRODERNEA (1999-2007). Trabajo y sociedad, 27, 447-473.

\section{Contribución de autoría}

Este trabajo fue realizado en partes iguales por Marcos Andrés Urcola y María Elena Nogueira. 